\title{
Research on CDMA2000 EV-DO Reverse Link Data Rate Control
}

\author{
Dai Mingjun \\ Dept. of Control Science and \\ Engineering, Harbin Institute of \\ Technology \\ Harbin Institute of Technology, 92 \\ West DaZhi Street, NanGang Dist, \\ Harbin, P.R.China 150001 \\ $+8613728646344$ \\ daimingjun3357@sina.com.cn
}

\begin{abstract}
This paper mainly studies on reverse link data rate control of CDMA2000 1x EV-DO system. We present the object of reverse link data rate control, aim at this object, combining practice and two basic prevailing control schemes, put forward a new control scheme. In this control scheme, implementation on AT side is described elaborately. First, we transfer the control object to mathematical model by explicitly design utility function form of some type of wireless data services, then, in order to adapt to EVDO system, we transform this model to an equivalent distributed model on AT side, after that, we utilize Lagrangian method to obtain solution of the distributed model, and prove that this solution satisfies the integrated problem's constraints put forward by the control object, we also design the MAC protocol based on this algorithm. At last, we validate availability and efficiency of the algorithm through experiment and simulation.
\end{abstract}

\section{Keywords}

Data rate control; utility; wireless network resources allocation.

\section{INTRODUCTION}

Wireless data business can offer rich and colorful services, such as video phones, video conference, wireless internet, electronic wallet, real-time online games, VoIP etc. These services will make communications between people more convenient and increase work efficiency as well. Many new practical data applications, which gradually change people's living habits, are being developed now, such as the mobile positioning and target searching service offered by Google, the popular MP3 downloading services in Europe. We can foresee that as more attractive applications being launched, wireless data communication will be more in depth to people's lives.

Along with the growing demand of wireless packet data services we mentioned above, more new wireless access technology will be introduced ceaselessly. The wireless access technology

Mobimedia 2008 July 7-9, 2008, Oulu, Finland. Copyright 2008 ICST ISBN 978-963-9799-25-7/08/07 DOI 10.4108/ICST.MOBIMEDIA2008. 4079

\author{
Dai Wangtao \\ Dept. of Control Science and \\ Engineering, Harbin Institute of \\ Technology \\ Harbin Institute of Technology, 92 \\ West DaZhi Street, NanGang Dist, \\ Harbin, P.R.China 150001 \\ $+8675589407833$ \\ daiwangtao2000@sina.com.cn
}

represented by wireless local access network can offer relatively high bandwidth, but the limitations in security, billing and coverage have restricted its wide application. In contrast, cellular mobile communication network can offer wide-area coverage, better billing system and security architecture, if combined with new innovated high-speed wireless access technology, it will have a bright future in offering wireless internet access services. One of the key design goals of the $3^{\text {rd }}$ generation mobile communication system is to provide a high-speed wireless data services. CDMA2000 1x EV-DO, one of 3G standards, is seen as wireless extension of internet. High-speed transmission is the core request of EV-DO system function, high-speed means based on limited bandwidth resources, provides mobile users with high-speed data services by using cellular mobile communication network, which is similar to cable network. Original purpose of designing EV-DO system is to provide one-way wireless Internet business, such as browse web and download files. Based on the characteristics of the service provided, original design goal is to provide non-realtime, dissymmetrical high-speed packet data services. At first, the keystone of system optimization design lies in forward link, while optimization of reverse link is less emphasized, that is to say the transmission speed and through-output of forward link must be higher than that of reverse link. As business develops, especially the increase of application demands, such as video conference, interactive games online and mobile storage, people begin to pay more attention to reverse link data rate.

Just like CDMA 1x system, EV-DO system still use the method of Code Division Multiple Access to communicate in reverse link, all users in the same cell (sector) transmit data at the same time and frequency, distinguished by different WALSH code modulation. As a result, users disturb each other when they transmit data in reverse link, we have to use reverse link power control technology to overcome Near-Far effects and make sure the system is not overloaded. The difference is that the data service provided by EV-DO system needs to meet users' requirements of data through-output. In HRPD protocol, AT (Access Terminal) can adjust speed of transmitting message by itself according to condition of wireless environment. The relationship between AT's transmitting data rate and power is shown in figure 1 . Herein, we take T2P, ratio of total power and pilot channel's power as vertical axis, we can get a meaningful mapping of rate and power. We can learn from the figure that T2P increases according to speed. As a result, it is important to restrict transmitting speed of AT and make sure the system is not overloaded. 


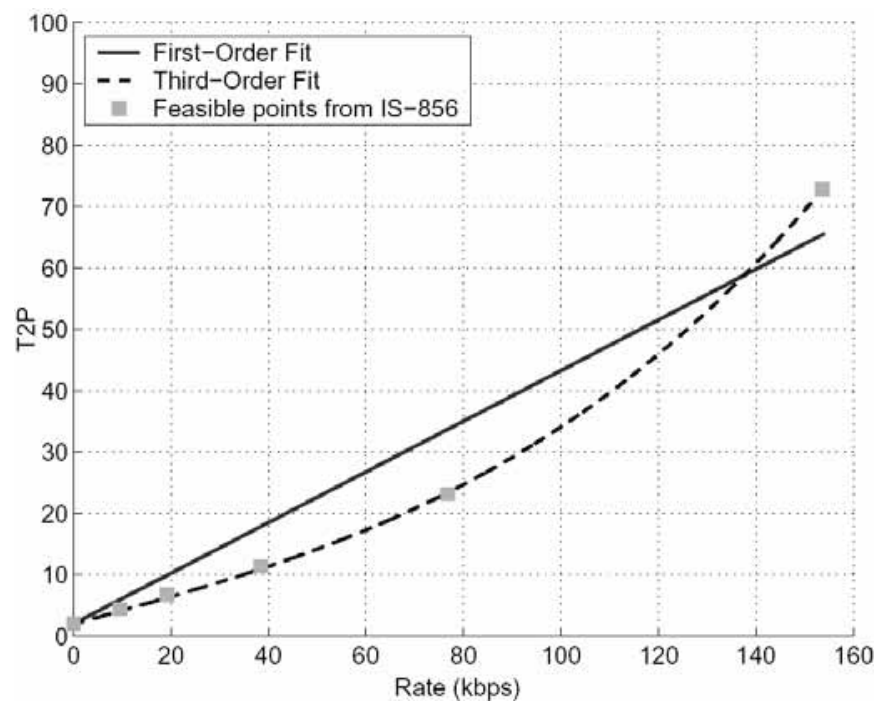

Figure1 T2P-Data rate mapping

In fact, reverse-link-data-rate control problem is a problem of allocation of network resources. With the spread of CDMA network, in order to solve the problem between customers' demands and low bandwidth, research on maximizing system utility function through designing CDMA system's resources allocation algorithm has been largely launched in many famous research centers and companies.

\section{LITERATURE REVIEW}

Earlier research just focused on resources allocation in fixed-line network. In 1998, professor F.Kelly in Cambridge published a paper "Rate Control in Communication Networks: Shadow prices, Proportional fairness and Stability”, put forward distributed computing allocation method based on market model in economics, he proved Lyapunov stability and fairness of this model to users, which laid foundation for further research. Though his work focused on ATM network, it can be used in CDMA wireless data communication network if some additional constraints were added to the method proposed in the paper. In the mean time, many famous companies including Lucent, Qualcomm, Motorola and Nokia all devoted into research on this project. Typical examples are rate control algorithm used in wireless LAN (IEEE 802.11) offered by Intel company according to Game Theory, and HRPD reverse link data rate control algorithm offered by Qualcomm. Wherein, HRPD Phase 0 uses transition probability matrix to implement control scheme, it can ensure maximizing utility function of the system and ensure fairness to all users in the long term, however, since it is based on probability which needs a relatively long time and large scale of ATs, if we view it in a relatively short time or small scale of area, the fairness is not very perfect. Acting as a random access scheme, WLAN's performance is rather unstable, especially as the network overload increases, through-output will increases to maximum value, however, with the continuing increase of network overload, the through-output of the network will decrease markedly. Thus, 802.11 MAC protocols can't maintain the network stay at the state of maximum through-output in long term.

This paper is going to overcome the above problems, it explicitly designs a utility function form that can fit for some wireless data services, which can satisfy fairness even in relatively short term and maximum utility and through-output of the system. The structure of this paper is as follows: firstly, based on the importance of reverse link data rate control, combining existing constraints, put forward the target we want to get; secondly, finish the modeling work, abstract the target into mathematical model and put it into equivalent distributed mathematical model based on Kelly's research results; thirdly, adopt Lagrangian method to get the solution of the distributed mathematical model, and discretize it in order to meet requirements of engineering application, we also prove convergence of the algorithm, which ensures one of the aim (fairness of every user) put forward in the beginning of this paper; lastly, use practical data to do simulation, which validate the effectiveness, stability, convergence.

\section{SYSTEM MODEL (CONTROL AIM)}

The significance of reverse link data rate control has been clarified in the beginning, combining with significance and practical running of system, the aims this paper is going to achieve will be put forward as follows:

\subsection{Aim 1: ensure system run in stability, no overload}

To ensure normal running of the system, we require the system having no overload. This point is the original intention of bringing forward reverse link data rate control. No overload means the power collected on AN (Access Network) side as the sum of all AT's power in every cell (sector) stays in a reasonable bound. As CDMA system is a self-interference system, any user's signal is viewed as interference for other users. Let RoT(Rise over Thermal) represent load of the system, $P_{i}$ represent user i's pilot power intensity, $T_{i}$ represent $T 2 P$ (ratio of the total transmit power to the pilot power), $N_{0}$ represent interference (including neighbour cell interference, deemed as constant), $N$ represent number of users in current cell (sector), a system's normal running requires:

$$
\operatorname{RoT}(t)=1+\sum_{i=1}^{N} \frac{T_{i}(t) P_{i}(t)}{N_{0}} \leq R o T_{t h}
$$

\subsection{Aim 2: aim of maximizing utility function}

Focus on using minimum cost (either resources the system shall allocate or payment the user has to pay for his service) to get in return maximum satisfaction. This paper only considers the scenario that user's satisfaction is proportional to data transfer rate, which can be represented by a strictly concave function. This supposition is fit for data services like ftp downloading (uploading) service, but not fit for services easily affected by time delay like VoIP, video conference, video telephone services etc.

\subsection{Aim 3: aim of maximizing utility function}

Since we can't satisfy providing every user with the same service quality at the same instant, as a commercialized communication system, we need to ensure fairness to every user. Then how to evaluate fairness? Assume in one period of time, if the average data rate is equal for every user that pays for the same payment for their services in the same cell (sector), then we think it satisfies requirements of fairness. Select time constant $\mathrm{m}$ as weighted average to represent average transmitted data rate 
$\rho_{i}(k)$ of $\mathrm{AT}_{\mathrm{i}}$ in a period of time, $R_{i}(k)$ as transmitted rate at instant i,

$$
\rho_{i}(k+1)=\frac{1}{m}\left[(m-1) \rho_{i}(k)+R_{i}(k)\right]
$$

Fairness can be seen as finding a converging way to let

$$
\lim _{k \rightarrow \infty} \rho_{i}(k)=c g_{i}, \forall i
$$

$g_{i}$ lies on the rank of data services that user selects.

\subsection{Aim 4: actually just a physical constraint}

Due to physical constraints, every AT's rate can't exceed a threshold. Thus, this constraint is,

$$
\begin{aligned}
& R_{i}(k)>0, i \in[1,2, \cdots, N] \\
& R_{i}(k)<R_{\text {max }}, i \in[1,2, \cdots, N]
\end{aligned}
$$

\section{FIX OF CONTROL SCHEME}

Feedback is soul of control, we adopt feedback to implement rate control. At present there are two basic modes of designing control scheme: integrated control mode and distributed control mode.

Integrated control means forming recorded information which keeps record of every user's status in this cell (sector) on the AN side through air interface to interact with every AT, based on the recorded information, AN can forecast quality of wireless environment, and allocate rate for every user. The advantage of this mode is that we can get an optimal result theoretically, and can implement QoS control over users. But, take practical application into account, there exist two disadvantages:

- Disadvantage 1: allocation algorithm needs multiple iteration, real-time performance can hardly be fulfilled. If placed on AN side, it is limited by embedded micro-processor's computing ability.

- Disadvantage 2: AT needs to interact with AN for more data to provide AN with enough information to deal with every AT separately, it also will cost lots of precious air-interface resources, largely impacting air interface and minimizing capacity of the system.

Distributed control means the control algorithm stays in every AT, the rate is controlled by every AT by itself. Advantages are:

- Advantage 1: can bring AT's computing ability into play, ensure quality of real-time.

- Advantage 2: the algorithm is simple relative to integrated algorithm, fast converging rate.

- Advantage 3: information interacted between AT and $\mathrm{AN}$ is little, thus causing small impact on the precious air-interface resources. AN doesn't need to allocate resources for every AT, saving AN's system resources.
Though theoretically performance of distributed control method is not as high as in integrated system, synthetically considering both advantages and disadvantages, and in order to be compatible with current protocols so as to enlarge our research results' probability of practical application, we decide to adopt integrated-control, distributed computation control scheme, the principle is as shown in figure 2 ,

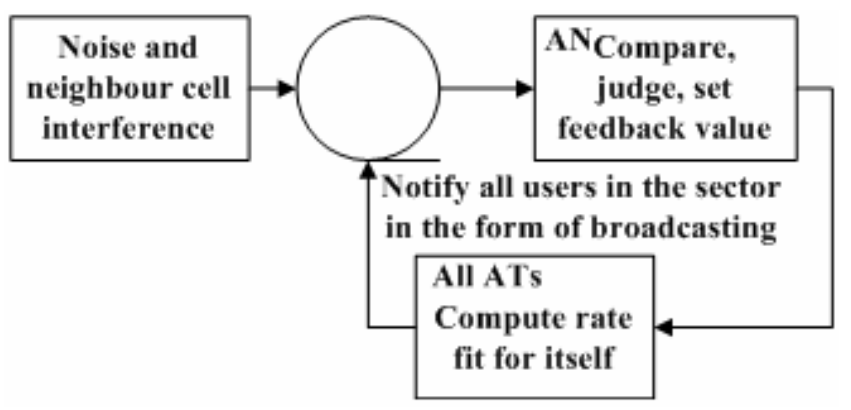

Figure 2 Control Scheme block diagram

On AN side, current status of system is computed according to the sum of all the AT's power and white noise and nieghbour cell interference, if it exceeds overloading threshold, then AN will feedback the information to all ATs. On AT side, this feedback information represents the price it should pay for the system's resources, i.e, the state of current system's resources' shortage, if the system is busy, cost of using high transmitting rate will naturally be high, AT will select an appropriate rate according to the condition (the average transfer rate before, if it had a little higher rate before, it should slow down relatively this time, prerequisite is these users select the same rank of service) of itself. Thus control scheme of integrated feedback to all ATs on AN side, and computes by itself on AT side is realized.

\section{IMPLEMENTATION OF INTEGRATED FEEDBACK ON AN SIDE}

The principle of integrated feedback control is relatively simple, let $R o T$ represent the load of the system, $I_{0}$ represent power spectrum intensity of the signal collected on AN side, $N_{0}$ represent intensity of noise power spectrum in the cell (sector), $R o T=\frac{I_{0}}{N_{0}}$. When RoT exceeds the preset threshold $R o T_{t h}$, the system is viewed as having reached the high limit of the system's capacity, then, AN should notify every AT the status (status of needy resources) of the current system through air-interface. Every AT adjusts its reverse link data transmitting rate separately according to the information given by $\mathrm{AN}$, so the aim of controlling the reverse link data rate is achieved.

\section{IMPLEMENTATION OF DISTRIBUTED COMPUTATION ON AT SIDE}

We first change Kelly's constraints on ATM network's distributed resources allocation algorithm to constraints fit for CDMA system, then design a time-varying utility function that can satisfy fairness, and abstract the reverse link data rate problem to a problem solving for maximum of utility function. Use Lagrangian method to design algorithm on AT side, prove convergence of the algorithm, validate that the algorithm can satisfy fairness 
requirements. Lastly, we validate that the algorithm can ensure system's normal running.

\subsection{Mathematical model of reverse link data rate control problem}

Herein, we establish mathematical model according to the control aim so as to find solution of the model and realize the goal for future. Suppose there are $N$ ATs in one cell (sector), let $P_{i 0}(k)$ represent pilot signal's power of $\mathrm{AT}_{\mathrm{i}}$, let $T_{i}(\mathrm{k})$ represent value of T2P for $\mathrm{AT}_{\mathrm{i}}$, we can get the total power of $\mathrm{AT}_{\mathrm{i}}$ as $P_{i}(\mathrm{k})=P_{i 0}(\mathrm{k})$ $T_{i}(k) . R_{i}(k)$ represent reverse link data transmitting rate of $\mathrm{AT}_{\mathrm{i}}, k$ represent the $k$ th data sub-frame in discrete time, as figure 1 shows, the relationship between $T_{i}(k)$ and $R_{i}(k)$ is one-to-one mapping, suppose

$$
\begin{aligned}
& T_{i}(k)=F_{R}\left(R_{i}(k)\right) \\
& R_{i}(k)=F_{T}\left(T_{i}(k)\right)
\end{aligned}
$$

We can get first-order fit results which is a linear function, while cubic fit result as a convex function.

For aim 1, let $Z(k)$ represent RoT, then we get equation (6)

$$
Z(k)=10 \log \left(1+\sum_{i=1}^{N} \frac{T_{i}(k) P_{\text {pilot }}}{N_{0} W}\right)
$$

Wherein, $P_{\text {pilot }}$ represents power intensity of AT's pilot signal, actually there always exists fluctuation in pilot power, but rarely come out that RoT is nominated by single AT's pilot power intensity, thus, fluctuation can be neglected. Let threshold of RoT be $Z_{t h}$, we need $Z(k)<Z_{t h}$, substitute (4), (6) into the above inequation, we get

$$
\sum_{i=1}^{N} F_{R}\left(R_{i}(k)\right) \leq \frac{N_{0} W}{P_{\text {pilot }}}\left(10^{\left.Z_{\text {th }} / 10_{-1}\right)}\right.
$$

Let $F_{R}\left(R_{i}(k)\right)=a R_{i}(k)+b, P_{t h}=\frac{N_{0} W}{P_{P i l o t}}\left(10^{Z_{t h} / 10}-1\right)-b N$, then aim 1 is equivalent to the following model,

$$
\sum_{j=1}^{N} F_{R}\left(R_{j}(k)\right) \leq P_{t h}
$$

For aim 2 and aim 3: maximizing utility function and ensure fairness. To achieve these goals, we adopt the concept "utility" introduced from economics field representing customer's satisfaction as an evaluation criterion of the system's outputinput-ratio.

Follow Kelly's method of solving problem of ATM network's resources allocation, we can view aim 2 as a problem of solving for maximum of utility function. Herein, we should design utility function's form, a concept first used in economics field, introduced to network research recently. Utility represents acquired customer's satisfaction that a customer accepts some type of service. Generally, utility function is monotonic, not decreasing, its preponderance lies in it essentially reflects customer's requirements, and also quantify customer's adaptability. Suppose data service is an elastic service, i.e, satisfaction of customer with the QoS provided by network system is evaluated by through-output of network system. Let $U_{i}\left(R_{i}\right)$ represent customer's utility function, $R_{i}$ as allocated transmitting rate for user $i$. The form of $U_{i}$ must be carefully selected to accurately reflect satisfaction extent. Generally speaking, $U_{i}(0)=0, U_{i}(\infty)=U_{\max }$, and $U_{i}$ works as $R_{i}$ 's monotonic, not decreasing function.

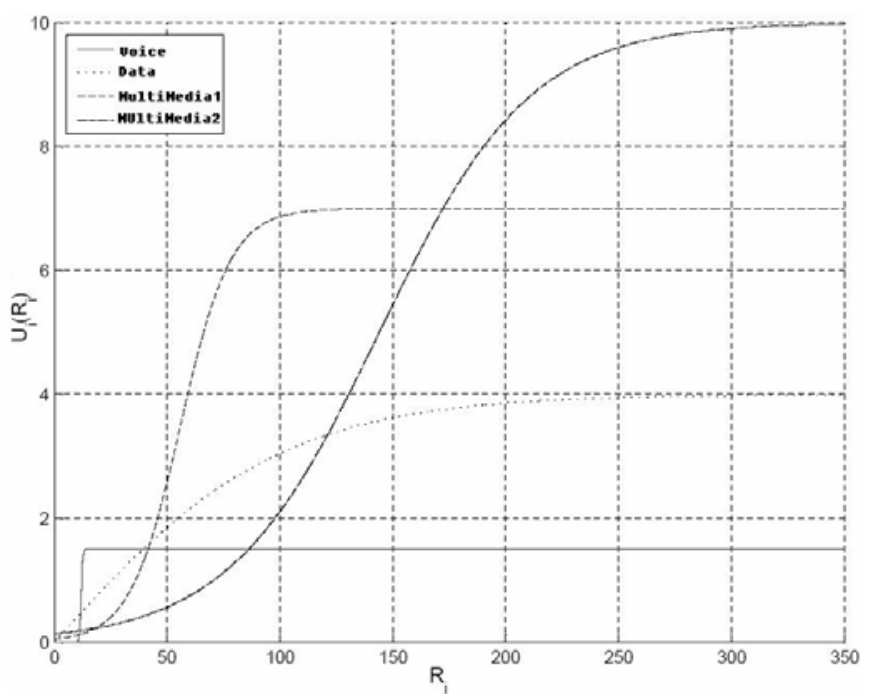

Figure 3 utility functions for different applications

From the figure above, we can get the conclusion that traditional voice business needs only clear voice in the call, so its utility function is similar to a step function; while for services like ftp downloading services, sometimes, customer will be more satisfactory if the rate is higher, and when the rate reaches a threshold (can satisfy customer's need), customer will not be sensitive to it any more; there are also other businesses like video conference, people's requirements on transferring of picture is similar to data business, while their requirements on voice is similar to voice business, so comes the curve like multimedia 1 and multimedia 2.

If we want to use utility function to express the relation between user's satisfaction, rate, and time, it should be increasing relative to rate, and increasing relative to $g_{i}$ (rank of service the user selects), meanwhile, utility function should consider fairness between users that select the same service, it should be monotonic and decreasing relative to $\rho_{i}(k)$, let $\lambda(\lambda \geq 0)$ represent sensitivity relative to $\rho_{i}(k)$, suppose utility function has the form as follows,

$$
U_{i}(R(k), k)=g_{i} \operatorname{In}\left(1+\frac{R_{i}(k)}{\lambda \rho_{i}(k)}\right)
$$

Thus, combining aim 1, 2, 3, 4, we can boil the reverse link data rate control problem down to a problem of solving for the solution of maximizing utility function, suppose linear form of $F\left(R_{\dot{i}}(k)\right)$

is $F_{R}\left(R_{i}(k)\right)=a R_{i}(k)+b . P_{t h}=\frac{N_{0} W}{P_{\text {Pilot }}}\left(10^{Z_{t h} / 10}-1\right)-b N$, then we get problem A as follows, i.e, concluded as a none-linear programming problem (NLP). 


$$
\begin{aligned}
& \max _{R(x)} \sum_{j=1}^{N} g_{j} \operatorname{In}\left(1+\frac{R_{j}(k)}{\lambda \rho_{j}(k)}\right) \\
& \text { s.t. } \sum_{j=1}^{N} F_{R}\left(R_{j}(k)\right) \leq P_{t h} \\
& R_{i}(k)>0, i \in[1,2, \cdots, N] \\
& R_{i}(k)<R_{\text {max }}, i \in[1,2, \cdots, N]
\end{aligned}
$$

\subsection{Design of algorithm on AT side}

We propose an algorithm based on time-varying utility function, validate that the algorithm can satisfy the control goal, the purpose of putting forward utility function is to give a solving method that can both ensure requirements of stability and fairness of system. The principle is as follows: firstly translate the problem of maximizing utility function on AN side into an equivalent problem, i.e, a problem of optimizing themselves on AT side separately, where we adopt Kelly's conclusion; then utilize Lagrangian's method to solve for the distributed optimization problem's solution, and validate it is also solution of maximizing utility function's problem on AN side.

As has been proved in Kelly's paper that optimization solution of the problem that satisfies every AT is also optimal solution on AN side for ATM system. Following this method, we properly translate constraints in ATM network into constraints fit for this paper, then solution of problem A is equivalent to solving problem of equation (11) that every AT can solve its own solution according to feedback $M(k)$ all by itself,

$$
\max _{R(k)} g_{i} \ln \left(1+\frac{R_{i}(k)}{\lambda \rho_{i}(k)}\right)-R_{i}(k) M(k)
$$

Wherein, $M(k)$ represents the price per unit rate. Then, we translate the system's problem into problem B of solving for optimal solution on each AT by itself through feedback.

For the sake of simplifying discussion, we list Lagrangian theorem which includes inequation constraints, i.e, the KKT (Karush-Kuhn-Tucker) condition.

Theorem 1: consider the following maximizing problem:

$$
P: \max f(x), x \in X
$$

Wherein, $f(x)$ is any given function, the constraints is

$$
h_{i}(x)=b, g_{j}(x) \leq 0
$$

$$
\begin{gathered}
\text { Construct } \\
L(x, \lambda)=f(x)+\lambda^{T}(b-h(x))+\mu^{T} g(x) \text { and aggrangian }
\end{gathered}
$$

$A(x)=\left\{j \in\{1,2, \ldots, r\} \mid g_{j}(x)=0\right\}$. Suppose $x^{*}$ is maximizing problem

(12)'s solution in the condition of constraints (13), then there exist $\lambda^{*} \in R^{m}$ and $\mu^{*} \in R^{r}$ satisfy

$$
\nabla_{X}\left(L\left(x^{*}, \lambda^{*}, \mu^{*}\right)\right)=\nabla f\left(x^{*}\right)+\sum \lambda_{i}^{*} \nabla h_{i}\left(x^{*}\right)+\sum \mu_{j}{ }^{*} \nabla g_{j}\left(x^{*}\right)=0
$$

And satisfy $\left\{\begin{array}{cc}\mu_{j}^{*} \geq 0 & \forall j=1, \ldots, r \\ \mu_{j}^{*}=0 & \forall j \notin A\left(x^{*}\right)\end{array}\right.$. Where $\nabla$ represent gradient.

Suppose $P_{t h}=P_{t h}-b N$, consider $R_{i}(k) \in\left[0, R_{\max }\right]$. Then we use Lagrangian method to validate theorem 2 , and then induce the method by theorem 2 .

Theorem 2: Let $\mathrm{R}_{\mathrm{i}}(\mathrm{k}) \in[0, \mathrm{Rmax}]$, and $F_{R}$ is linear function, if feedback $M(k)$ is the least value that satisfy (14)

$$
\sum_{j=1}^{N}\left(\frac{g_{i}}{M(k)}-\lambda \rho_{i}(k)\right) \leq \frac{P_{t h}}{a}
$$

Then we get equation (10) and (11)'s solution are both

$$
R_{i}(k)=\frac{g_{i}}{M(k)}-\lambda \rho_{i}(k)
$$

Proof: Firstly, it's easy to validate that (15) is solution of (11). The following is to validate it is also solution of (10), (10)'s Lagrangian function is as follows,

$L(R(k), \gamma, \mu, \sigma)=\sum_{j=1}^{N} g_{i} \ln \left(1+\frac{R_{j}(k)}{\lambda \rho_{j}(k)}\right)-\gamma\left(\sum_{j=1}^{N} a R_{j}(k)-P_{t h}\right)+\sum_{j=1}^{N} \mu_{j} R_{j}-\sum_{j=1}^{N} \sigma_{i}\left(R_{j}-R_{\max }\right)$

Do partial differential computation, and let it be 0 , we get

$$
\frac{\partial L}{\partial R_{i}(k)}=\frac{g_{i}}{R_{i}(k)+\lambda \rho_{i}(k)}-a \gamma+\mu_{i}-\sigma_{i}=0
$$

Where, for $\forall i, \gamma \geq 0, \mu_{i} \geq 0, \sigma_{i} \geq 0$.

So, solution $R_{i}(\mathrm{k})$ has the following form

$$
R_{i}(k)=\frac{g_{i}}{M(k)}-\lambda \rho_{i}(k)
$$

And $\mathrm{R}_{\mathrm{i}}(\mathrm{k}) \in[0, \mathrm{Rmax}], M(k)$ is the minimum value that satisfy (14). We can view the relation between variables from the solution as follows: rate $R_{i}(k)$ that each user can select is proportional to their payment (rank of service paid by users) $g_{i}$; is inverse proportional to its average rate before, i.e, it should lower its rate if it had a relatively high rate before, we can't let user maintain a high rate all the time relative to others if they select the same rank of service, thus reflects our algorithm's fairness; is inverse proportional to current system's resource's shortage status, i.e, if resources are currently in shortage which means the system is busy, in other words, the price per unit resources should be a little higher, then transmitting rate we select should be a little lower, otherwise, we have to pay relatively high payment. (14) illuminates that the minimum value means the payment is the least under condition of no-overloading of system and getting the maximum output, which can satisfy user's QoS requirements.

We can get the following algorithm from the above conclusion:

Step 1: when $\mathrm{k}=1, \mathrm{~g}_{\mathrm{i}}$ and $\rho_{i}(1)$ are both known to all $\mathrm{AT}_{\mathrm{i}} \mathrm{s}$ and $\mathrm{AN}$ in the system.

Step 2: first compute for the minimum $M(k)$ that satisfy (14), put it in broadcasting message in forward control channel to inform every AT. 
Step 3: after AT received $M(k)$, computes $R_{i}(k)$ following (15) separately, and notifies AN its next packet frame's format through the reverse channel.

Step 4: AN updates $\rho_{i}(k)$ after demodulating the reverse information from AT.

Step 5: repeat the above operation.

\subsection{Validation of AT algorithm's stability}

We will illuminate the algorithm can assure the system's stable running through simulation in this section. The simulation block diagram is as follows,

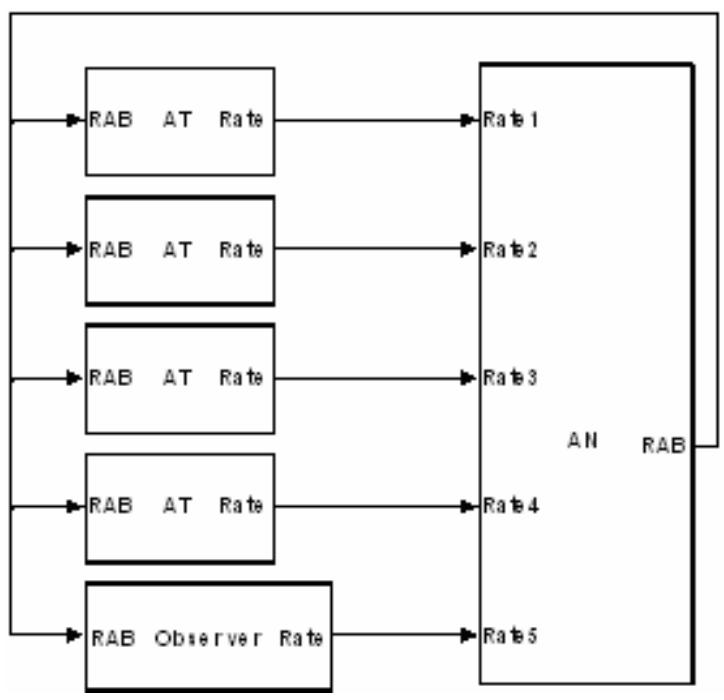

Figure 4 Block diagram of simulation fundamental

Set $\lambda=1, m=50$, we can get $a=0.4128, b=2$ from figure 1 . Set the threshold of Rot to be $P_{t h}=172$. We can see from the next simulation results that when 30 ATs access to system in random, we can satisfy stability requirement of system. Figure 6 represents one of the AT's allocated rate with respect to time, we can see any AT's rate can get to stable state in less than 10 steps which is very short time, thus each AT maintains stable.

The following T2P curve (figure 5) is obtained by computation with practical data. Where, AT accesses to the system randomly, $g_{i}$ is selected in the range regulated by protocol. We can see the T2P can stay relatively stable.

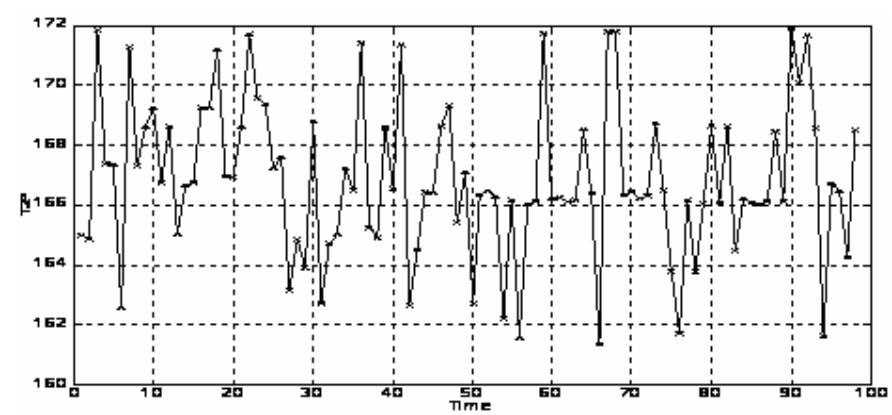

Figure 5 Curve of T2P relative to time

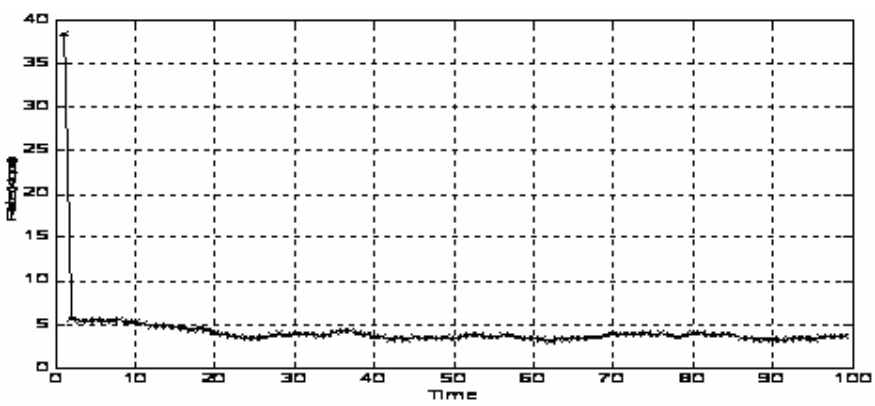

Figure 6 Rate curve relative to time

\section{CONCLUSION}

This paper does a research on reverse link data rate control tactic for CDMA2000 EV-DO system. It illuminates elaborately every detail from specification of control aim, to modeling of aim, to put-forward of control scheme, to implementation of algorithm on AN side and AT side, to prove of convergence of the algorithm and simulation validation. Based on the above research, we obtain an effective reverse link data rate control scheme. It is a not bad reference for controlling rate in CDMA2000 EV-DO and other network's rate.

Though wireless data business has shown a bright future, there also exist lots of problems. Such as the problem of how to efficiently specify threshold of RoT has not been solved; there also exist some deficiency in the control algorithm on AT side, which is based on supposition that mapping based on T2P and rate is linear, which can only applies to services like ftp-based downloading business, compatibility with current protocols is aslo not validated, converging rate is also not taken into account, how to provide QoS control over inter-users is not solved etc. The problem of reverse link data rate control is also the hotspot in fields of academia and industry.

\section{ACKNOWLEDGMENTS}

Our thanks to ACM SIGCHI for allowing us to modify templates they had developed.

\section{REFERENCES}

[1] F. Kelly, A. Maullb, and D. Tan, "Rate Control in Communication Networks: Shadow Prices, Proportional Fairness and Stability", Journal of the Operational Research Society, pp. 237-252, 1998.

[2] R. Padovani, "Reverse Link Performance of IS-95 Based Cellular Systems”, IEEE Personal Communication, 1994, pp. 28-34.

[3] Q. Wu and E. Esteves, The CDMA2000 High Rate Packet Data System, Qualcomm White Paper, 2002.

[4] F. Kelly, "Charging and Rate Control for Elastic Traffic", European Trans Telecomm, vol 8, 1998, pp. 33-37.

[5] S. Hanly, "Capacity and Power Control in Spread Spectrum Macro-diversity Radio Networks”, IEEE Transactions on Communications, vol. 44(2), 1996, pp. 247-256.

[6] D. Kim, K. Chang, and S. Sim, "Efficient Distributed Data Rate Control for Cellular Mobile Systems”, IEEE 
Transactions on Vehicular Technology, Vol. 46(5), pp. 313319.

[7] Y.K. Xiao, "Performance Research on the MAC Protocol and TCP in Wireless Ad Hoc Networks", Doctor Degree Dissertation Submitted to Tsing Hua University, 2004, pp. 27-59.
[8] Z. S. Niu, "Utility-Based Radio Resource Optimization for Multimedia DS-CDMA Systems”, Acta Electronic Sinica, 2004, vol(32) No. 10, pp. 1594-1599. 